\title{
Determination of sample size for two stage sequential designs in bioequivalence studies under $2 \times 2$ crossover design
}

\author{
Haile Mekonnen Fenta \\ Department of Statistics, College of Science, P.O. Box 79, Bahir Dar University, Bahir Dar, Ethiopia
}

Email address:

hailemekonnen@gmail.com

To cite this article:

Haile Mekonnen Fenta. Determination of Sample Size for Two Stage Sequential Designs in Bioequivalence Studies under 2x2 Crossover Design. Science Journal of Clinical Medicine. Vol. 3, No. 5, 2014, pp. 82-90. doi: 10.11648/j.sjcm.20140305.12

\begin{abstract}
Sequential design is an adaptive design that allows for pre-mature termination of a trial due to efficacy or futility based on the interim analyses. The concept of sequential statistical methods was originally motivated by the need to obtain clinical benefits under certain economic constraints. That is for a trial for a positive results, early stopping ensures that a new drug product can exploited sooner, while negative results indicated, early stopping avoids wastage of resources. In short, the right drug at the right time for the right patient. Furthermore, the possible implication of two stage sequential design/ sample size re-estimation is to adjust the sample size based on the observed variance estimated from the first stage. The purpose of this work was to determine the minimum number of sample size required to proceed the second stage of sequential design, and the simulation is done through $\mathrm{R}$ ve. 3.0.3 Statistical software package. In general, from our simulation study, we can understand that, for highly variable drugs $(\mathrm{CV} \geq 30)$, the appropriate GMR value is between $(0.95$, 1.05), which is also appropriate for low variable drugs to achieve the minimum sample size required to conduct any clinical trials.
\end{abstract}

Keywords: Two Stage Sequential Design, Geometric Mean Ratio, Bioequivalence Study, Power and Sample Size

\section{Introduction}

The sequential approach has been a natural way to proceed throughout the history of experimentation. Perhaps the earliest proponent was Noah, who on successive days released a dove from the Ark in order to test for the presence of dry land during the subsidence of the Flood [1]. Sequential design is an adaptive design this allows for pre mature termination of a trial due to efficacy or futility, based on the interim analyses. That is the right drug at the right time for the right patient. In general; Sequential methods typically lead to savings in sample-size, time, and cost when compared with the classic design with a fixed sample-size. The main purpose of sequential design is that, if the investigated active substance is known to have adverse effects, it may be necessary to use patients instead under suitable precautions and supervision. And the two drugs are said to be average bioequivalence (ABE) for second stage of sequential design,if and only if the confidence interval $(1-2 \alpha) \times 100 \%$ for the ratio of test to reference formulation is contained within the regulatory limits of $(\theta 1, \theta 2)$, specifically according to some regulatory agencies, like Food and Drug Administration's (FDA) 0.81.25 , or $-0.2231436-0.2231436$ for both $\mathrm{AUC}$ and $\mathrm{C}_{\max }$. A principal reasoning for conducting a group sequential test is discussed in detail in [2] and its aim is simply to decrease the sample size of the study units under two stage sequential designs.

A sample size re-estimation (SSR) refers to an adaptive design that allows for sample size adjustment or resampling based on the review of interim analyses results. The sample size requirements for the trial are sensitive to the effect size and its variability [3]. That is inaccurate estimation of the effect size and its variability leads overpowered or underpowered results, neither of which is desirable. If a trial is underpowered, when the variance used in the power calculation is too low or the chosen effect size overly optimistic, it will not be able to detect a clinically meaningful difference, and consciously prevent a potentially effective drug from being delivered to patients. On the other hand, if the trial is overpowered, it could lead to unnecessary exposure of many patients to a potentially 
harmful compound when the drug, in fact, is not effective [4].

The application of group sequential approaches to the BE studies differs from their application to most other types of clinical studies because the former generally involves crossover designs, testing of equivalence hypotheses, and testing based on t-distributions, whereas the later generally involves parallel designs with testing of difference hypotheses [5].At the $\mathrm{i}^{\text {th }}$ stage of a group sequential BE trial, data are analyzed from the first $\left(n_{i}\right)$ of planned maximum number of subjects $\mathrm{n}$, and the trial is stopped and $\mathrm{BE}$ is concluded if and only if the $(1-2 \alpha \mathrm{i}) \times 100 \% \mathrm{CI}$ for the test to reference ratios are entirely contained within the interval $\left[80,125 \%\right.$ ]for both $\mathrm{C}_{\max }$ (maximum drug concentration) and (the area under the drug concentration verses curve [6]. AUC is often used to measure the extent of absorption or the total amount of drug absorbed in the body). Otherwise the trial continues to the second stage [7].

\section{Methods}

\subsection{Two Stage Design}

First initial group of subjects are treated and data are analyzed, then if bioequivalence are not demonstrated, an additional subject can be employed, and the results from both groups combine for final statistical analyses [7, 8]. In general, two stage group sequential design with interim look after $n_{1}$ subject's complete and final look after $\left(n=n_{1}+n_{2}\right)$ subjects complete. Here we have the following potential decisions.

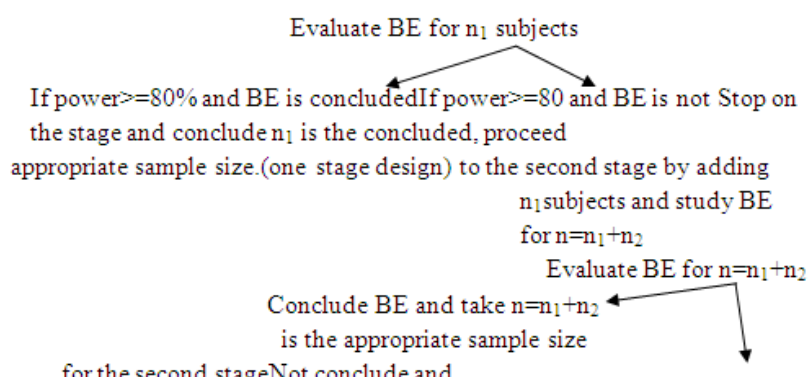

stop the study here.

Figure 1. two stage sequential design

More generally, the two stagesfor this design can be summarizedas follows:

1. In stage one (for $n_{1}$ subjects), after analyzing the given data, we decide to:

a. Stop and claim bioequivalence.

b. Continue the trial in second stage for $\left(n=n_{1}+n_{2}\right)$ subjects.

2. In stage two (for $n=n_{1}+n_{2}$ subjects)to:

a. Stop and claim bioequivalence.

b. Stop and do not claim bioequivalence.

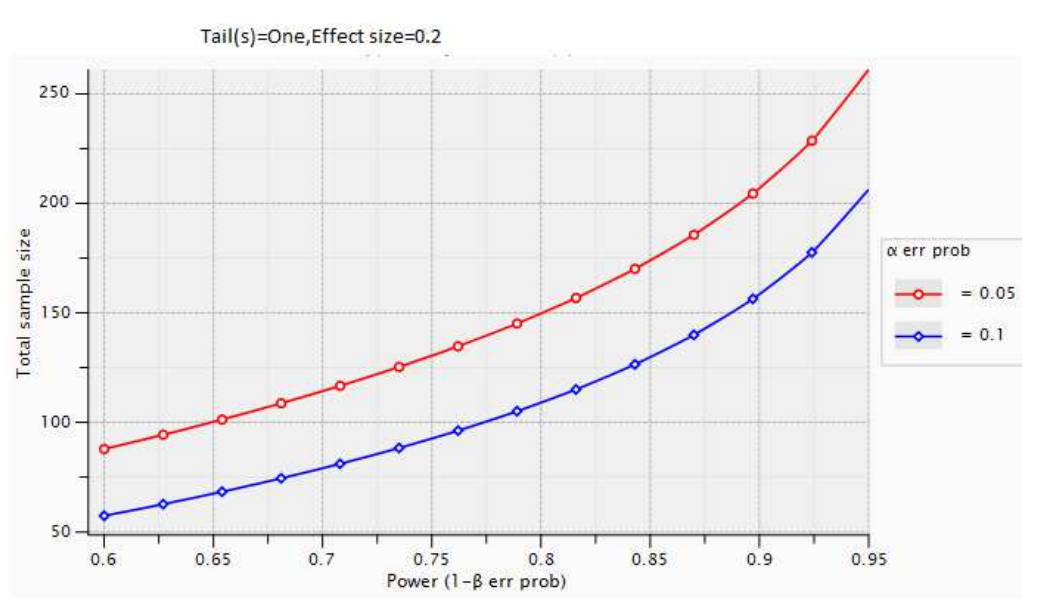

Figure 2. The relationship between type one error, power and sample size.

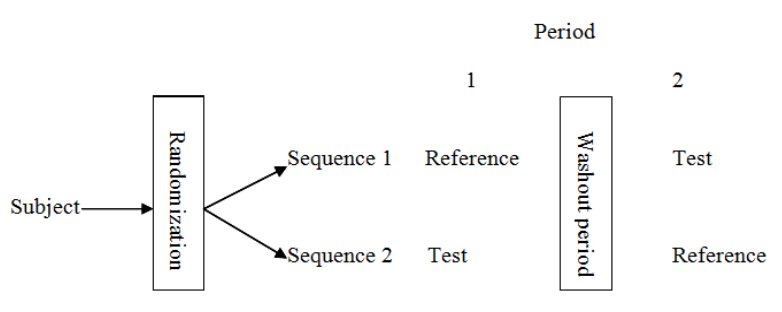

Figure 3. The simple $2 \times 2$ crossover design

The minimum sample size for stage two is 2 (if the decision rule determined that the study should continue to stage 2) and there is no upper limit to the size of stage 2 . This can be expressed as: Sample size for stage 2 is $[2, \infty)$ and here equal sample size assumption is also under consideration.

Consider a $2 \times 2$ crossover trial where we wish to compare reference $(\mathrm{R})$ and test $(\mathrm{T})$ using two sequences of treatment (RT and TR) given in two periods $[9,10]$. Let $\mathrm{n}_{1}$ and $\mathrm{n}_{2}$ subjects be allocated to the two sequences, respectively. Two statistical approaches are suggested in literature for testing bioequivalence between $\mathrm{T}$ and $\mathrm{R}$. These are:

Two One Sided Hypothesis Tests (TOST) procedure at $\alpha$ significance level and Let $\theta_{\mathrm{L}}$ and $\theta_{\mathrm{U}}$ are respectively lower 
and upper known clinically meaningful bioequivalence limits and $\theta$ be the parameter of interest $[11,12.13]$.

In TOST procedure, two sided bioequivalence test is divided in to two one-sided tests in the following manner:

$$
\begin{gathered}
\text { Test1: } \mathrm{H}_{\mathrm{o}}^{+}: \theta \leq \theta L \text { versus } \mathrm{H}^{+}: \theta>\theta L \\
\text { Test1: } \mathrm{H}_{\mathrm{o}}^{-}: \theta L \geq \theta \text { versus } \mathrm{H}_{1}^{-} \theta<\theta L
\end{gathered}
$$

(1-2 2$) \times 100 \%$ Confidence Interval Procedure: During the planning stage of bioavailability (BA) and bioequivalence (BE) study, the clinicians and the statisticians are able to answer the following questions [14, 15, 16, 17, and 18]. How many subjects are needed in order to achieve a desired power (commonly 80\%) to establish BE between two formulations within clinically/may not be statistically important limits $( \pm 20 \%$ of the reference mean)? If only small number of subjects is available in hand due to limited resources/budget or some medical considerations, what do we have to do? In order to answer the above critical questions, a statistical approach for sample size determination is employed $[17,18]$.

\subsection{Simulation Methodology and Formulas}

Both lower and upper confidence intervals are simulated based on the following formulas. And the proportion of the simulated confidence interval contained within $(0.8,1.25)$ is computed. Let $\mathrm{L}_{1}$ and $\mathrm{U}_{1}$ be the lower and upper intervals, respectively.

$$
\begin{aligned}
& \mathrm{L} 1=\frac{\bar{X} T}{\bar{X} R}-\mathrm{t}(\alpha, n 1+n 2-2) \hat{\sigma} d \sqrt{\frac{1}{n 1}+\frac{1}{n 2}} \\
& \mathrm{U} 1=\frac{\bar{X} T}{\bar{X} R}+\mathrm{t}(\alpha, n 1+n 2-2) \hat{\sigma} d \sqrt{\frac{1}{n 1}+\frac{1}{n 2}}
\end{aligned}
$$

Additionally, the power was calculated based on the modification of [7].

$$
\begin{gathered}
1-\beta=\mathrm{F}_{\mathrm{t}}\left(\frac{\ln (1.25 / \theta)}{s \sqrt{2 / n}}-\mathrm{t} 1-\alpha, \mathrm{df}, \mathrm{df}\right)-\mathrm{F}_{\mathrm{t}}\left(\frac{-\ln (1.25 / \theta)}{s \sqrt{2 / n}}-\mathrm{t} 1-\right. \\
\alpha, \mathrm{df}, \mathrm{df})
\end{gathered}
$$

where; $1-\beta$ is the power, $\mathrm{df}$ is the degrees of freedom associated with the error, $\operatorname{theF}_{\mathrm{t}}(\mathrm{x}, \mathrm{df})$ is the cumulative distribution functions of students' t-distribution with DF degrees of freedom, and lastly, $t_{1-\alpha}, D F$ is the $(1-\alpha)^{\text {th }}$ percentile of a student's t-density function. "s" is the sample standard deviation (estimate of $\sigma$ ) which is calculated from ANOVA on the $\ln ($ Test $/$ Reference $)=\ln ($ Test $)$ - $\ln$ (Reference) differences using stage/sequence, and stage*sequence effects in the model[3]. And finally, $\operatorname{GMR}(\theta)$ is the ratio of Test mean and reference mean of the two drug products.If the proportion of the confidence interval is greater than or equal to (1$2 \alpha) \times 100 \%$ and power is at least $80 \%, \mathrm{BE}$ is concluded and the corresponding sample size can be determined $[1,9]$. Generally; the simulation was performed using statistical software R. A different randomly selected seed was used for each scenario. A scenario was defined as a specified

\begin{tabular}{|c|c|c|c|c|c|}
\hline$\mu_{T}$ & $\mu_{R}$ & $\theta=\frac{\mu_{T}}{\mu_{R}}$ & $\begin{array}{l}\text { Sample } \\
\text { size (n) }\end{array}$ & $\sigma_{e}^{2}$ & $\mathbf{C V} \%=\frac{\sigma_{R}}{\mu_{R}} \times 100 \%$ \\
\hline 85 & \multirow{12}{*}{100} & 0.85 & 12 & 100 & 10 \\
\hline 90 & & 0.90 & 16 & 200 & 15 \\
\hline 95 & & 0.95 & 20 & 400 & 20 \\
\hline 100 & & 1.00 & . & 580 & 25 \\
\hline 105 & & 1.05 & . & 850 & 30 \\
\hline 110 & & 1.10 & . & 1200 & 35 \\
\hline 115 & & 1.15 & 100 & 1600 & 40 \\
\hline \multirow[t]{5}{*}{120} & & 1.20 & 110 & 2000 & 45 \\
\hline & & & 120 & 2500 & 50 \\
\hline & & & 130 & 3000 & 55 \\
\hline & & & 140 & & \\
\hline & & & 150 & & \\
\hline
\end{tabular}
combination of ratio of geometric means, intra-subject coefficient of variation (CV), and sample size. The given parameters and assumptions in the simulation work are given in the following table.

Table 1. The given parameters and assumptions in the simulation work

$$
\mu_{\mathrm{T}}=\text { The true test mean }
$$

$\mu_{\mathrm{R}}=$ The true references mean which is constant.

$\theta=$ Geometric mean ratio, thus, the equivalence limits for the difference are -20 to +20 and for the ratio are 0.8 and 1.25 .

$$
\sigma_{\mathrm{e}}^{2}=\text { Intra-subject variability }
$$

And for a sample size $n$,

Test $\sim \mathrm{N}\left(\mu_{\mathrm{T}}, \sigma_{\mathrm{T}}\right)$ and Reference $\sim \mathrm{N}\left(\mu_{\mathrm{R}}, \sigma_{\mathrm{R}}\right)$

Where, $\mathrm{n}, \mu, \sigma$ are, respectively, the sample size, the true mean and the true standard deviation for the test and the reference drug products. One million simulated studies were performed at $\alpha=0.05$ significant level. Note that, in our simulation results, the missing value is likely to be produced sometimes, if it is the case, it is replaced by the arithmetic mean of the other simulated data produced in each step. For all stages we evaluate BE based on the power approach for the given coefficient of variation $(\mathrm{CV})$ and geometric mean ratio (GMR). Let us summarize this in the following figure. Note, for any clinical trial; a minimum of 12-sample size is required to conduct a study.

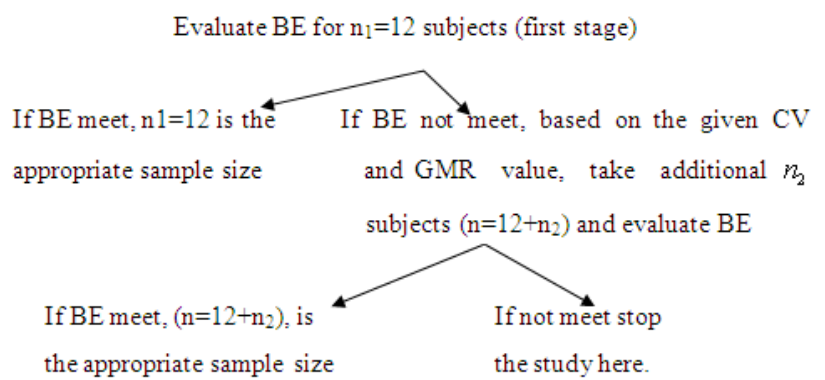

Figure 4. Simple schematic presentation of simulation for determining sample size for $B E$

\section{Results and Discussions}

For $\alpha=0.05$, the proportion of the simulated value for all approaches should include at least $90 \%$ to conclude BE and the corresponding sample size to be sufficient, which was the aim of this work. Since the minimum sample size required to conduct any clinical study is 12 , which is also 
the initial sample size in our simulation study, 6 , for each group.

For the given coefficient of variation and geometric mean ratios, the sample size determined from the simulation results is summarized in table 1 as follows.

Table 2. Summary of sample size for the second stage are summarized from simulation results as follows

\begin{tabular}{lllllllll}
\hline \multicolumn{1}{l}{ GMR $=\boldsymbol{\mu T} / \boldsymbol{\mu} \mathbf{R}$} \\
\hline $\mathbf{C V} \%$ & $\mathbf{0 . 8 5}$ & $\mathbf{0 . 9 0}$ & $\mathbf{0 . 9 5}$ & $\mathbf{1 . 0}$ & $\mathbf{1 . 0 5}$ & $\mathbf{1 . 1 0}$ & $\mathbf{1 . 1 5}$ & $\mathbf{1 . 2 0}$ \\
\hline 10 & 52 & 8 & 0 & 0 & 0 & 0 & 12 & 88 \\
15 & 60 & 12 & 0 & 0 & 0 & 8 & 8 & \\
20 & 108 & 24 & 4 & 4 & 8 & 36 & 98 & \\
25 & & 36 & 12 & 8 & 28 & 60 & & \\
30 & & 60 & 24 & 28 & 60 & & & \\
35 & & 98 & 40 & 40 & & & & \\
40 & & & 76 & 76 & & & & \\
45 & & & 118 & & & & & \\
50 & & & & & & & & \\
55 & & & & & & & & \\
\hline
\end{tabular}

As shown from the simulation results in table 2 above, we can observe the required sample size needed to conduct a clinical trial when the $G M R=0.85$ with the corresponding $\mathrm{CV}$ values. As a result when the $\mathrm{GMR}=0.85$ and $\mathrm{CV}=10$, we need additional 52 subjects, in addition to the initial sample size $n=12$. While for second stage, $C V=15$, additional 60 subjects are required to demonstrate $\mathrm{BE}$ $(n=12+60)$. However, generally, we can observe that when $\mathrm{CV}$ increases from 10 to 15 ,we need additional 8 subjects, 4 for each group. When the coefficient of variation increases from 10to 20, the sample size extremely increases to 108.Finally, for this GMR value there is no need of conducting any clinical trial for $\mathrm{CV}>20$.

The summary of sample size for GMR $=0.90$ is also given in table 2 above and specifically, when the $G M R=0.90$, and $\mathrm{CV}=10$, additional 8 subjects 4 , subjects for each group, are important to achieve $\mathrm{BE}$ between the test and the reference drug products. For second stage, when $\mathrm{CV}=15$, additional 12 samples are needed $(n=12+12)$. From the results shown in the table, we can understand that for $\mathrm{GMR}=0.90$ and $\mathrm{CV}=20$, only 24 in addition to the initial 12 subjects, which is almost one third of the sample size required in case of $\mathrm{GMR}=0.85$ for a constant $\mathrm{CV}$ values are needed to demonstrate BE. This implies that the sample size is highly affected by GMR, in addition to $\mathrm{CV}$ values. For $\mathrm{GMR}=0.9$, for small values of $(\mathrm{CV}<30)$, a maximum of 48 subjects/ sample size is needed. In addition, for high values of $\mathrm{CV} \geq 30$, a minimum of 72 subjects are needed to conduct a clinical study. However, when CV values $(>35)$, proceeding to the next step/stage is not important. It may be harmful both ethically and economically as illustrated in the introduction part of this paper. Let us see the results when the $G M R=0.95$. The minimum number of sample size is achieved compared to the above two GMR values for a constant $\mathrm{CV}$. Even, for $\mathrm{CV}=10$ and $15, \mathrm{BE}$ is achieved in the first stage which was not possible for the above two GMR values. For example, for $\mathrm{CV}=20$, only 4 subjects are needed, 2 for each group. And here we go for other CV values similarly. To summarize, from the table given above, for small values of $\mathrm{CV}$ a maximum of 24 subjects are required, and for high values of $\mathrm{CV}$, at least 36 subjects are needed. And for $\mathrm{CV}>40$, we have to stop the study to protect wastage of resources.

When the $G M R=1.0$, and $\mathrm{CV}$ value is small, the simulation result shows similar sample size value with that of $G M R=0.95$. In addition, we stop the study when $\mathrm{CV}>40$, which is the critical value of $\mathrm{CV}$ with the corresponding sample size $=88$. Nevertheless, for small values of $\mathrm{CV}$, this GMR value is more appropriate in terms of sample size determinations than $\mathrm{GMR}=0.95$. For example, when $\mathrm{CV}=25,36$ and 12 sample sizes are required to conduct a clinical trial for $\mathrm{GMR}=0.95$ and 1.0 , respectively.

When $G M R=1.05$, as we can see from table 2 above the simulation result shows that, for small values of $\mathrm{CV}$, almost similar results of sample size is required with that of $G M R=0.95$, and 1.0. While for large values of $\mathrm{CV}$, adding additional sample size is unimportant. In other words, we have to stop the study here and must use other alternatives. Specifically, for GMR 0.9 and 1.05, as we can see from table 2 above, for $\mathrm{GMR}=0.9$ and for small values of $\mathrm{CV}$ more number of sample size is needed compared to $\mathrm{GMR}=1.05$, while for large values of $\mathrm{CV}$ the reverse is true. In general, from the simulation results we can observe that, in order to determine the appropriate values of sample size, we have to see both GMR and CV values carefully.

And when the $G M R=1.10$, for small values of $\mathrm{CV}$, the simulation result shows, approximately two times the number of subjects needed for $G M R=0.95,1.0$, and 1.05. However, when the variance becomes more heterogeneous, like $\mathrm{CV}>30$ it is unimportant to conduct any trial for the given $\mathrm{GMR}=1.10$; it is unethical as well as wastage of time and resource. For example, for $\mathrm{CV}=25$, it is important to add $60(72=12+60)$ additional number of sample size to conduct $\mathrm{BE}$ study for the second stage. For $G M R=1.15$, the simulation result in table 2shows that based on the $\mathrm{CV}$ values, for the second stage, additional 12,32 and 98 subjects are required in addition to the 12 initial subjects. For example, when $\mathrm{CV}=10$ a total of $24(12+12)$ samples is required, but we have 12 , which is the initial sample size for any clinical trial as we stated before. So, additional 12's are important.For simplicity, when $\mathrm{CV}$ is 20 , we have to take additional 98 subjects/sample size in addition to the first 12.Finally, for $\mathrm{GMR}=1.20$, for $\mathrm{CV}=10$, the sample size is $\mathrm{n}=100$, that is additional 88 samples are important. However, for $\mathrm{CV}>10$, there is no need of taking additional sample size to conduct $\mathrm{BE}$ trials.

\subsection{Summaries and Recommendations of the Study}

In general, from our simulation study shown in table 2 above, we can understand that, for highly variable drugs $(\mathrm{CV} \geq 30)$, the appropriate GMR value is between $(0.95$, $1.05)$, which is also appropriate for low variable drugs to achieve the minimum sample size required to conduct any clinical trials. For GMR values less than 0.95 and more than 1.05 , we need a maximum number of subjects even for 
low variable drug products. Finally, from our simulation result given in the appendix, we can observe that when the sample size increases, the proportion of $(1-2 \alpha) \times 100 \%$ Confidence interval contained in $(0.8,1.25)$ highly increases, even for large values of $\mathrm{CV}$ and any values of GMR, but the value of the power, which is very important to detect meaningful clinical difference decreases. As a result, based on the power approach, demonstrating BE and determining the corresponding sample size for highly variable drugs and for GMR values out of the range $(0.95$, $1.05)$, is very difficult. Moreover, the other important thing here is that, as the intra-subject coefficient of variation (CV) increases, the power decreases and larger sample sizes are needed to achieve a given power. As a conclusion, from our simulation results we observed that, the appropriate GMR to conduct $\mathrm{BE}$ study is $(0.95,1.05)$, which is also recommended in FDA.

N.B: In the appendix part, the simulation result for samples $(n=40,44,48,52,56,60,64,68,72,76,80,84,88$, $92,96,100$ and 110) are not displayed in order to save the number of pages.

\section{Acknowledgements}

I would like to thank Prof. Dr. Mehmet N Orman, department head of Biostatistics and Bioinformatics, Ege University/Turkey/Izmir, for his advice and willingness to share his insightful thoughts with me, which was very fruitful for shaping up my ideas and the entire work.

\section{Appendix}

Simulation Results

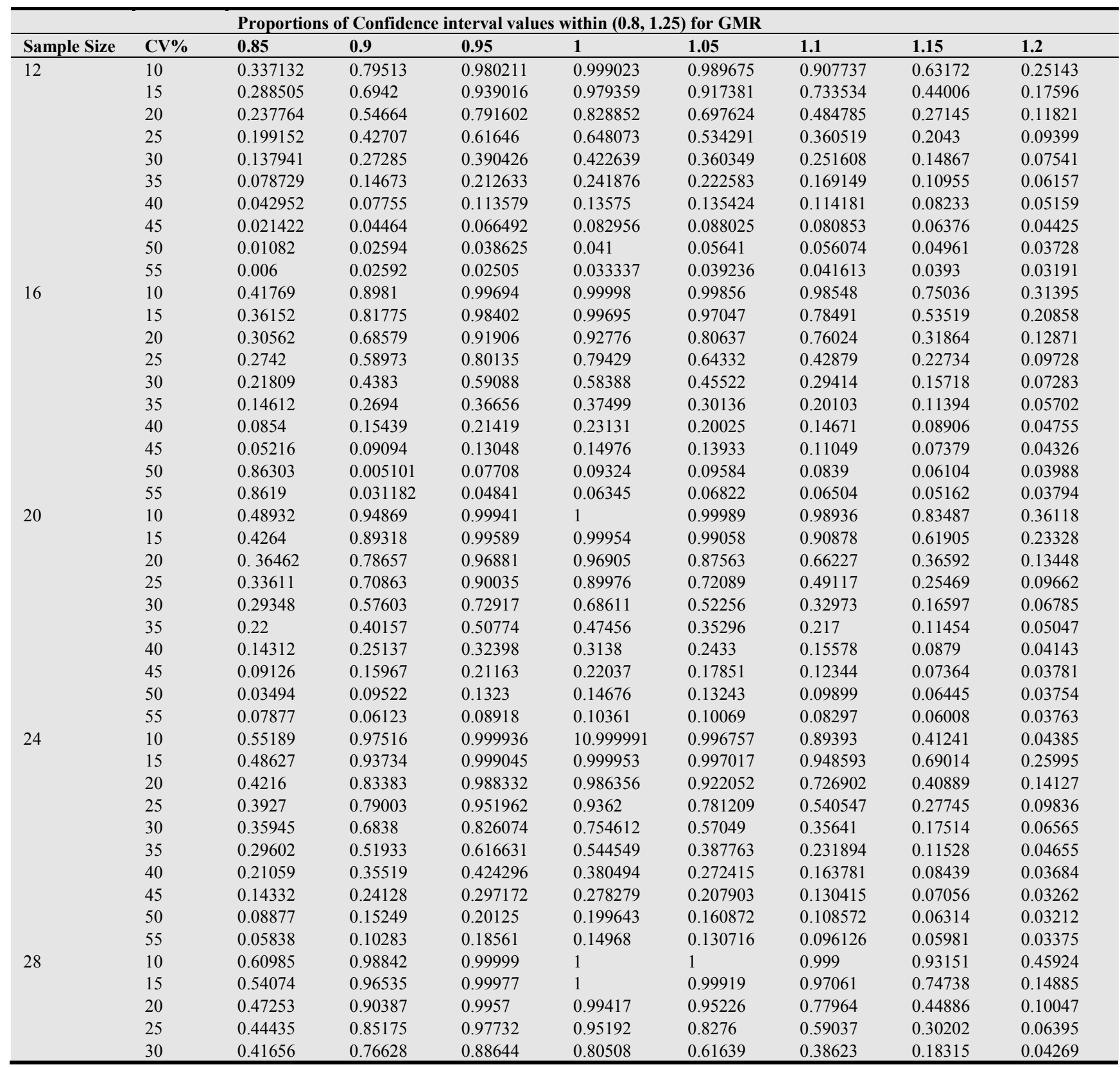




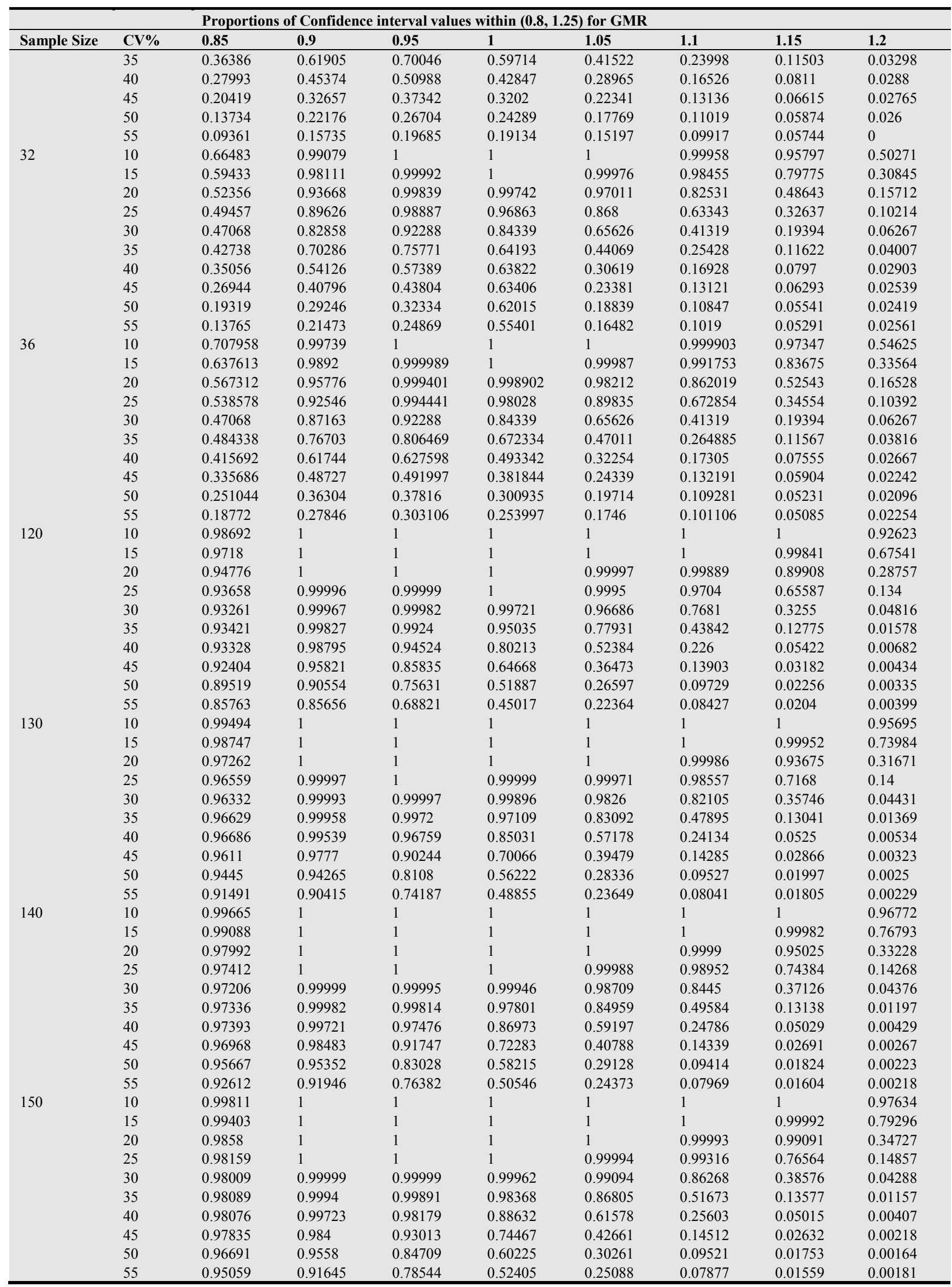


Continued to Simulation Results

\begin{tabular}{|c|c|c|c|c|c|c|c|c|c|}
\hline \multirow[b]{2}{*}{ Sample Size } & \multirow[b]{2}{*}{ CV\% } & \multicolumn{8}{|c|}{ Corresponding power values for all values of mean ratio (GMR) } \\
\hline & & 0.85 & 0.9 & 0.95 & 1 & 1.05 & 1.1 & 1.15 & $1.20=(G M R)$ \\
\hline \multirow[t]{10}{*}{12} & 10 & 0.337132 & 0.56249 & 0.980211 & 0.999023 & 0.989675 & 0.907737 & 0.63172 & 0.25143 \\
\hline & 15 & 0.009471 & 0.26495 & 0.980722 & 0.998079 & 0.992461 & 0.844657 & 0.22483 & 0.00154 \\
\hline & 20 & 0.002156 & 0.06563 & 0.841966 & 0.950614 & 0.888476 & 0.492769 & 0.05747 & 0.00015 \\
\hline & 25 & 0.000307 & 0.002389 & 0.436652 & 0.633913 & 0.488147 & 0.140131 & 0.00774 & $1.00 \mathrm{E}-05$ \\
\hline & 30 & $9.00 \mathrm{E}-05$ & 0.00703 & 0.22278 & 0.375481 & 0.25279 & 0.052589 & 0.00208 & $1.00 \mathrm{E}-05$ \\
\hline & 35 & $8.00 \mathrm{E}-05$ & 0.00221 & 0.089261 & 0.171257 & 0.102133 & 0.015858 & 0.00073 & 0 \\
\hline & 40 & $5.00 \mathrm{E}-06$ & 0.00227 & 0.033896 & 0.071018 & 0.038863 & 0.004897 & 0.00015 & 0 \\
\hline & 45 & $1.00 \mathrm{E}-06$ & 0.00042 & 0.014677 & 0.032707 & 0.016765 & 0.001948 & $4.00 \mathrm{E}-05$ & 0 \\
\hline & 50 & 0 & 0.00037 & 0.004971 & 0.012 & 0.005353 & $6.00 \mathrm{E}-04$ & 0 & 0 \\
\hline & 55 & 0 & 0.00032 & 0.003308 & 0.007886 & 0.003838 & 0.000428 & 0 & 0 \\
\hline \multirow[t]{10}{*}{16} & 10 & 0.01318 & 0.85141 & 0.99694 & 1 & 0.99998 & 0.98546 & 0.44059 & 0.00136 \\
\hline & 15 & 0.0022 & 0.50486 & 0.98402 & 0.99794 & 0.9912 & 0.78368 & 0.11077 & $1.00 \mathrm{E}-05$ \\
\hline & 20 & 0.00014 & 0.12521 & 0.91906 & 0.8791 & 0.76352 & 0.27329 & 0.01046 & 0 \\
\hline & 25 & $2.00 \mathrm{E}-05$ & 0.03821 & 0.40993 & 0.62199 & 0.64567 & 0.09368 & 0.00196 & 0 \\
\hline & 30 & 0 & 0.00875 & 0.16369 & 0.30804 & 0.18558 & 0.02369 & 0.00026 & 0 \\
\hline & 35 & 0 & 0.00187 & 0.05552 & 0.12366 & 0.06451 & 0.00536 & $7.00 \mathrm{E}-05$ & 0 \\
\hline & 40 & 0 & 0.00061 & 0.02019 & 0.0513 & 0.02428 & 0.00165 & $2.00 \mathrm{E}-05$ & 0 \\
\hline & 45 & 0 & 0.00026 & 0.0097 & 0.02574 & 0.01151 & 0.00073 & $1.00 \mathrm{E}-05$ & 0 \\
\hline & 50 & 0 & 0.00012 & 0.00489 & 0.01346 & 0.00611 & 0.00028 & $1.00 \mathrm{E}-05$ & 0 \\
\hline & 55 & 0 & $6.00 \mathrm{E}-05$ & 0.00291 & 0.00897 & 0.00393 & 0.00016 & 0 & 0 \\
\hline \multirow[t]{10}{*}{20} & 10 & 0.02289 & 0.97568 & 1 & 1 & 1 & 0.99971 & 0.70234 & 0.000134 \\
\hline & 15 & 0.00289 & 0.7571 & 0.99931 & 0.99999 & 0.99972 & 0.95005 & 0.21705 & $3.00 \mathrm{E}-05$ \\
\hline & 20 & 0.00013 & 0.23392 & 0.90237 & 0.97442 & 0.92576 & 0.46772 & 0.01658 & 0 \\
\hline & 25 & $1.00 \mathrm{E}-05$ & 0.07019 & 0.63045 & 0.82361 & 0.67898 & 0.1737 & 0.00024 & 0 \\
\hline & 30 & 0 & 0.01374 & 0.28196 & 0.48554 & 0.31975 & 0.03858 & 0 & 0 \\
\hline & 35 & 0 & 0.00267 & 0.09389 & 0.20612 & 0.10731 & 0.0078 & 0 & 0 \\
\hline & 40 & 0 & 0.00063 & 0.03211 & 0.08147 & 0.03708 & 0.00191 & 0 & 0 \\
\hline & 45 & 0 & 0.00023 & 0.01402 & 0.03909 & 0.01611 & 0.00065 & 0 & 0 \\
\hline & 50 & 0 & 0.00013 & 0.00657 & 0.01901 & 0.00029 & 0 & 0 & 0 \\
\hline & 55 & 0 & $8.00 \mathrm{E}-05$ & 0.00387 & 0.01143 & 0.00396 & 0.00021 & 0 & 0 \\
\hline \multirow[t]{10}{*}{24} & 10 & 0.99852 & 1 & 1 & 1 & 0.999999 & 0.89465 & 0.00242 & \\
\hline & 15 & 0.0451 & 0.92375 & 0.999979 & 1 & 0.999995 & 0.993572 & 0.37952 & $4.00 \mathrm{E}-05$ \\
\hline & 20 & $8.00 \mathrm{E}-05$ & 0.39902 & 0.975339 & 0.995362 & 0.982824 & 0.678704 & 0.03043 & 0 \\
\hline & 25 & 0 & 0.12569 & 0.808073 & 0.995363 & 0.840534 & 0.295894 & 0.00387 & 0 \\
\hline & 30 & 0 & 0.02316 & 0.432194 & 0.653015 & 0.473073 & 0.06907 & 0.00028 & 0 \\
\hline & 35 & 0 & 0.00367 & 0.154681 & 0.313356 & 0.176845 & 0.01234 & $3.00 \mathrm{E}-05$ & 0 \\
\hline & 40 & 0 & 0.00073 & 0.051792 & 0.130473 & 0.060602 & 0.00762 & 0 & 0 \\
\hline & 45 & 0 & 0.00019 & 0.020824 & 0.060623 & 0.024726 & 0.002512 & 0 & 0 \\
\hline & 50 & 0 & $7.00 \mathrm{E}-05$ & 0.028617 & 0.010787 & 0.000825 & 0.000293 & 0 & 0 \\
\hline & 55 & 0 & $3.00 \mathrm{E}-05$ & 0.016423 & 0.005839 & 0.000293 & 0.000146 & 0 & 0 \\
\hline \multirow[t]{10}{*}{28} & 10 & 0.08418 & 0.99996 & 1 & 1 & 1 & 1 & 0.97824 & 0.00408 \\
\hline & 15 & 0.00784 & 0.98559 & 1 & 1 & 1 & 0.99967 & 0.58388 & $3.00 \mathrm{E}-05$ \\
\hline & 20 & 0.00018 & 0.59061 & 0.99456 & 0.99913 & 0.99656 & 0.84323 & 0.05511 & 0 \\
\hline & 25 & $1.00 \mathrm{E}-05$ & 0.21803 & 0.91387 & 0.97445 & 0.92995 & 0.45261 & 0.00625 & 0 \\
\hline & 30 & 0 & 0.04015 & 0.58908 & 0.78471 & 0.62521 & 0.11671 & 0.00035 & 0 \\
\hline & 35 & 0 & 0.00567 & 0.23487 & 0.4354 & 0.26392 & 0.01964 & 0 & 0 \\
\hline & 40 & 0 & 0.00074 & 0.08049 & 0.19403 & 0.09431 & 0.00381 & 0 & 0 \\
\hline & 45 & 0 & 0.00021 & 0.03176 & 0.09033 & 0.03782 & 0.00099 & 0 & 0 \\
\hline & 50 & 0 & $9.00 \mathrm{E}-05$ & 0.01346 & 0.0427 & 0.015 & 0.00023 & 0 & 0 \\
\hline & 55 & 0 & $3.00 \mathrm{E}-05$ & 0.00698 & 0.02349 & 0.00745 & 0.00015 & 0 & 0 \\
\hline \multirow[t]{10}{*}{32} & 10 & 0.15689 & 1 & 1 & 1 & 1 & 1 & 0.99724 & 0.00742 \\
\hline & 15 & 0.01451 & 0.99817 & 1 & 1 & 1 & 1 & 0.77113 & $8.00 \mathrm{E}-05$ \\
\hline & 20 & 0.00018 & 0.76157 & 0.99878 & 0.9997 & 0.99913 & 0.93714 & 0.10002 & 0 \\
\hline & 25 & $1.00 \mathrm{E}-05$ & 0.33786 & 0.963 & 0.98898 & 0.97078 & 0.61272 & 0.01109 & 0 \\
\hline & 30 & 0 & 0.06656 & 0.71805 & 0.87095 & 0.74882 & 0.18586 & 0.00062 & 0 \\
\hline & 35 & 0 & 0.00852 & 0.33091 & 0.55203 & 0.36734 & 0.03269 & $2.00 \mathrm{E}-05$ & 0 \\
\hline & 40 & 0 & 0.00122 & 0.12074 & 0.55089 & 0.13811 & 0.00552 & $1.00 \mathrm{E}-05$ & 0 \\
\hline & 45 & 0 & 0.00033 & 0.04781 & 0.55076 & 0.05629 & 0.00139 & $1.00 \mathrm{E}-05$ & 0 \\
\hline & 50 & 0 & $6.00 \mathrm{E}-05$ & 0.01907 & 0.5321 & 0.02242 & $3.00 \mathrm{E}-04$ & 0 & 0 \\
\hline & 55 & 0 & $4.00 \mathrm{E}-05$ & 0.00905 & 0.25664 & 0.01174 & 0.00017 & 0 & 0 \\
\hline \multirow[t]{3}{*}{36} & 10 & 0.263753 & 1 & 1 & 1 & 1 & 1 & 0.99977 & 0.01315 \\
\hline & 15 & 0.027538 & 0.99983 & 1 & 1 & 1 & 0.999997 & 0.90215 & $7.00 \mathrm{E}-05$ \\
\hline & 20 & 0.000346 & 0.88541 & 0.999578 & 0.999873 & 0.99974 & 0.978222 & 0.17142 & 0 \\
\hline
\end{tabular}




\begin{tabular}{|c|c|c|c|c|c|c|c|c|c|}
\hline \multirow[b]{2}{*}{ Sample Size } & \multirow[b]{2}{*}{$\mathrm{CV} \%$} & \multicolumn{8}{|c|}{ Corresponding power values for all values of mean ratio (GMR) } \\
\hline & & 0.85 & 0.9 & 0.95 & 1 & 1.05 & 1.1 & 1.15 & $1.20=($ GMR $)$ \\
\hline \multirow{17}{*}{120} & 25 & $5.00 \mathrm{E}-06$ & 0.48738 & 0.984359 & 0.995091 & 0.98727 & 0.7531 & 0.02005 & 0 \\
\hline & 30 & 0 & 0.11288 & 0.71805 & 0.87095 & 0.74882 & 0.18586 & 0.00062 & 0 \\
\hline & 35 & 0 & 0.01438 & 0.437992 & 0.656194 & 0.47434 & 0.052066 & $6.00 \mathrm{E}-05$ & 0 \\
\hline & 40 & 0 & 0.00181 & 0.173676 & 0.352129 & 0.19769 & 0.008747 & $3.00 \mathrm{E}-05$ & 0 \\
\hline & 45 & 0 & 0.00031 & 0.071183 & 0.182467 & 0.08216 & 0.002095 & $1.00 \mathrm{E}-05$ & 0 \\
\hline & 50 & 0 & 0.00011 & 0.027972 & 0.087753 & 0.03328 & 0.000542 & 0 & 0 \\
\hline & 55 & 0 & $4.00 \mathrm{E}-05$ & 0.013599 & 0.048259 & 0.01604 & 0.000185 & 0 & 0 \\
\hline & 10 & 1 & 1 & 1 & 1 & 1 & 1 & 1 & 1 \\
\hline & 15 & 1 & 1 & 1 & 1 & 1 & 1 & 1 & 0.8349 \\
\hline & 20 & 0.88763 & 1 & 1 & 1 & 1 & 1 & 0.99992 & 0.00133 \\
\hline & 25 & 0.17437 & 0.99969 & 0.99997 & 0.99999 & 1 & 0.99991 & 0.99313 & 0 \\
\hline & 30 & 0.00126 & 0.9905 & 0.99957 & 0.99989 & 0.99956 & 0.99555 & 0.71564 & 0 \\
\hline & 35 & 0 & 0.87882 & 0.99655 & 0.99873 & 0.99659 & 0.94746 & 0.09856 & 0 \\
\hline & 40 & 0 & 0.51437 & 0.97904 & 0.99415 & 0.98274 & 0.73053 & 0.00316 & 0 \\
\hline & 45 & 0 & 0.20538 & 0.93169 & 0.97986 & 0.9399 & 0.42967 & 0.00011 & 0 \\
\hline & 50 & 0 & 0.05494 & 0.82774 & 0.94182 & 0.84636 & 0.18307 & $1.00 \mathrm{E}-05$ & 0 \\
\hline & 55 & 0 & 0.01648 & 0.70057 & 0.89119 & 0.73147 & 0.07527 & 0 & 0 \\
\hline \multirow[t]{10}{*}{130} & 10 & 1 & 1 & 1 & 1 & 1 & 1 & 1 & 1 \\
\hline & 15 & 1 & 1 & 1 & 1 & 1 & 1 & 1 & 0.8349 \\
\hline & 20 & 0.88763 & 1 & 1 & 1 & 1 & 1 & 0.99992 & 0.00133 \\
\hline & 25 & 0.17437 & 0.99975 & 0.99997 & 0.99999 & 1 & 0.99991 & 0.99313 & 0 \\
\hline & 30 & 0.00126 & 0.99428 & 0.99957 & 0.99989 & 0.99956 & 0.99555 & 0.71564 & 0 \\
\hline & 35 & 0 & 0.92166 & 0.99655 & 0.99873 & 0.99659 & 0.94746 & 0.09856 & 0 \\
\hline & 40 & 0 & 0.61774 & 0.97904 & 0.99415 & 0.98274 & 0.73053 & 0.00316 & 0 \\
\hline & 45 & 0 & 0.2914 & 0.93169 & 0.97986 & 0.9399 & 0.42967 & 0.00011 & 0 \\
\hline & 50 & 0 & 0.09072 & 0.82774 & 0.94182 & 0.84636 & 0.18307 & $1.00 \mathrm{E}-05$ & 0 \\
\hline & 55 & 0 & 0.03002 & 0.78881 & 0.93485 & 0.81055 & 0.11801 & 0 & 0 \\
\hline \multirow[t]{10}{*}{140} & 10 & 1 & 1 & 1 & 1 & 1 & 1 & 1 & 1 \\
\hline & 15 & 1 & 1 & 1 & 1 & 1 & 1 & 1 & 0.94943 \\
\hline & 20 & 0.96354 & 1 & 1 & 1 & 1 & 0.99999 & 0.99996 & 0.00045 \\
\hline & 25 & 0.31707 & 0.99983 & 1 & 1 & 1 & 0.99994 & 0.99633 & 0 \\
\hline & 30 & 0.00352 & 0.99653 & 0.99978 & 0.99994 & 0.99998 & 0.99737 & 0.81752 & 0 \\
\hline & 35 & $1.00 \mathrm{E}-05$ & 0.94908 & 0.99798 & 0.99944 & 0.99974 & 0.96708 & 0.16339 & 0 \\
\hline & 40 & 0 & 0.71167 & 0.98869 & 0.99684 & 0.99009 & 0.80704 & 0.00739 & 0 \\
\hline & 45 & 0 & 0.38156 & 0.95874 & 0.99 & 0.96393 & 0.53232 & 0.00031 & 0 \\
\hline & 50 & 0 & 0.13776 & 0.88588 & 0.96851 & 0.89943 & 0.25537 & $1.00 \mathrm{E}-05$ & 0 \\
\hline & 55 & 0 & 0.04811 & 0.70057 & 0.89119 & 0.73147 & 0.07527 & 0 & 0 \\
\hline \multirow[t]{10}{*}{150} & 10 & 1 & 1 & 1 & 1 & 1 & 1 & 1 & 1 \\
\hline & 15 & 1 & 1 & 1 & 1 & 1 & 1 & 1 & 0.99864 \\
\hline & 20 & 0.99759 & 1 & 1 & 1 & 1 & 1 & 0.99999 & 0.03543 \\
\hline & 25 & 0.66565 & 0.99987 & 0.99999 & 1 & 1 & 0.99997 & 0.9983 & $1.00 \mathrm{E}-05$ \\
\hline & 30 & 0.02197 & 0.99657 & 0.99995 & 0.99998 & & 0.9989 & 0.92682 & 0 \\
\hline & 35 & $2.00 \mathrm{E}-05$ & 0.94788 & 0.99944 & 0.9999 & 0.99995 & 0.98662 & 0.34984 & 0 \\
\hline & 40 & 0 & 0.71414 & 0.99663 & 0.99916 & 0.99958 & 0.90667 & 0.02793 & 0 \\
\hline & 45 & 0 & 0.38166 & 0.9861 & 0.99721 & 0.99689 & 0.71191 & 0.0014 & 0 \\
\hline & 50 & 0 & 0.13744 & 0.9566 & 0.97882 & 0.98886 & 0.43301 & $6.00 \mathrm{E}-05$ & 0 \\
\hline & 55 & 0 & 0.04854 & 0.90774 & 0.95321 & 0.96278 & 0.24025 & 0 & 0 \\
\hline
\end{tabular}

\section{References}

[1] Phillips, K.F. power of the two-one sided tests procedure in bioequivalence study. J.pharmacokin.biopharm;1990. 18(7), 137-144.

[2] Jones, B. Bioequivalence and statistics in clinical pharmacology. $1^{\text {st }}$ ed. Chapman \& Hall/crc: Boca raton; 2006.

[3] Potvin, et. equential design approach for bioequivalence studies with crossover designs. Pharmaceutical statistics;2008. 7(17), 245-262.

[4] Deletti, E., Hauschke, D., \& Steinjans, V.W.. Sample size determination for bioequivalence assessment by means of confidence intervals. Int. J.clin. Pharm. Ther. Toxicol; 1991.29(7), 1-8.

[5] Grizzle, J.E. the two-period cross-over design and its use in clinical trials: biometrics; 1965. 21(13), 467-480.

[6] Hauck, W.W., Preston, P.E., \& Bois, F.Y. a group sequential approach to crossover trials for average bioequivalence. Journal of biopharmaceutical statistics;1997.7(9), 87-96.

[7] Pocock S. Group sequential methods in the design and analysis of clinical trials. Biometrika;1977. 64 (8),191-199.

[8] Bonate, P.L. \& Howard, D, R. Pharmacokinetics in drug development.3rd ed. Springer, heidelberg dordrecht london 2011. 
[9] Liu, J. P. \& Weng, C.Sevaluation of parametric and nonparametric two one-sided tests procedures for assessing bioequivalence of average bioavailability. Journal of biopharmaceutical statistics.1993.3(17), 85-102.

[10] Schuirmann, D.. comparison of the two one-sided tests procedure and the power approach for assessing the equivalence of average bioavailability. Journal of pharmacokinetics and bio-pharmaceutics; 1987.15(13), 657-680.

[11] Altman, D. G. Statistics in medical journals. Statistics in medicine; 1982. 1:59-71.

[12] Chow, S.C., \& Liu, J.P.. Design and analysis of bioavailability and bioequivalence studies.3rd edn. Chapman \& Hall/crc: Boca raton; 2009.
[13] O'brien, P.C., \& Fleming, T.R. a multiple testing procedure for clinical trials. Biometrika; 1979. 35(7), 549-556

[14] Patterson, S., \&Jones, B. Bioequivalence and statistics in clinical pharmacology. Chapman \& hall/crc: boca raton; 2006.

[15] Westlake, W.J. the use of confidence intervals in comparative bioavailability trials. Journal of pharmaceutical sciences; 1972. 61(1), 1340-1341.

[16] Chow, S.C. adaptive design methods in clinical trials. Chapman \& Hall/crc: Boca raton; 2007.

[17] Julious, s. A. Sample sizes for clinical trials. Chapman \& Hall/crc, Boca raton; 2010.

[18] Senn, S. Crossover trials in clinical research. $2^{\text {nd }} e d$. Charater: john wiley \& sons; 2002. 